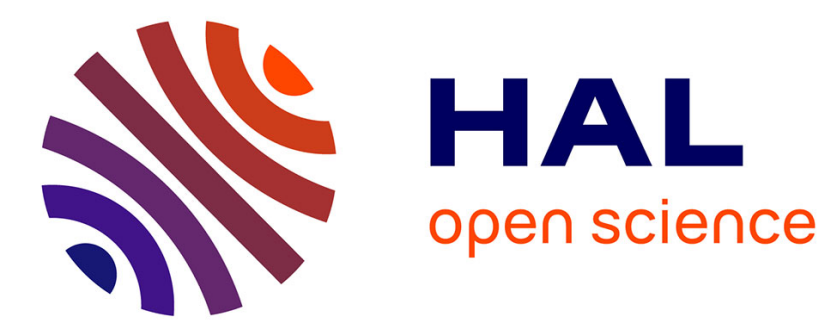

\title{
Star-based a posteriori error estimates for elliptic problems.
}

Boujemaa Achchab, Abdellatif Agouzal, Naima Debit, Khalid Bouihat

\section{To cite this version:}

Boujemaa Achchab, Abdellatif Agouzal, Naima Debit, Khalid Bouihat. Star-based a posteriori error estimates for elliptic problems.. Journal of Scientific Computing, 2014, 60 (1), pp.184-202. 10.1007/s10915-013-9793-x . hal-00690013

\section{HAL Id: hal-00690013 https://hal.science/hal-00690013}

Submitted on 20 Apr 2012

HAL is a multi-disciplinary open access archive for the deposit and dissemination of scientific research documents, whether they are published or not. The documents may come from teaching and research institutions in France or abroad, or from public or private research centers.
L'archive ouverte pluridisciplinaire HAL, est destinée au dépôt et à la diffusion de documents scientifiques de niveau recherche, publiés ou non, émanant des établissements d'enseignement et de recherche français ou étrangers, des laboratoires publics ou privés. 


\title{
Star-based a posteriori error estimates for elliptic problems
}

\author{
B. Achchab ${ }^{a}$, A. Agouzal ${ }^{b}$, N. Debit ${ }^{b, 1}$ and K. Bouihat ${ }^{a}$ \\ ${ }^{a}$ Université Hassan $1^{e r}$, $\mathrm{LM}^{2} \mathrm{CE}$, Ecole Supérieure de Technologie de Berrechid \\ et Faculté des Sciences Economiques, Juridiques et Sociales Settat, B.P 218 Berrechid, Maroc. \\ achchab@yahoo.fr, khalidbouihat@yahoo.fr \\ ${ }^{b}$ Université de Lyon; CNRS ; Université Lyon 1 ; Institut Camille Jordan, \\ F-69622 Villeurbanne cedex, France. \\ agouzal@univ-lyon1.fr, naima.debit@univ-lyon1.fr
}

\begin{abstract}
We give an a posteriori error estimator for nonconforming finite element approximations of diffusionreaction and Stokes problems, which relies on the solution of local problems on stars. It is proved to be equivalent to the energy error up to a data oscillation, without requiring Helmholtz decomposition of the error nor saturation assumption. Numerical experiments illustrate the good behavior and efficiency of this estimator for generic elliptic problems.
\end{abstract}

Key words: A posteriori error estimator, nonconforming finite element method, diffusion reaction equations, Stokes equations.

AMS subject classification: 65D05, 65D15, 65N50.

\section{Introduction}

During the last two decades a large amount of work has been devoted to a posteriori error estimation for solution approximated either by conforming $[1,17]$ or nonconforming $[2,8,9]$ finite element methods. In the nonconforming context, two main approaches have been considered for constructing an a posteriori error estimator. In residual estimators some extra terms have to be added to well-known a posteriori error estimator used in conforming framework. In $[3,4,10]$, these extra terms are the jumps across the element edges of the tangential derivatives of the finite element approximation with respect to element edges.

One of the most successful estimators proposed by Bank and Weiser and extended by many authors $([1,6,7,12])$, is based on the solution of local Neumann problems on elements, which seems to allow for cancellation and yields better effectivity indices than residual estimators in numerical tests performed in [11]. The classical proof of equivalence with the energy error require the saturation assumption which states that this solution can be approximated asymptotically better with quadratic than with linear finite elements. The saturation assumption is shown to be superfluous by Nochetto in [13]. However, removing this assumption requires comparison with residual estimators. More recently, an approach based on the solution of local problems on stars was proposed in [11, 15], and the proof of the equivalence with energy error applies directly without reference to residual estimators. This approach is applied in

\footnotetext{
${ }^{1}$ Corresponding author. E-mail: naima.debit@univ-lyon1.fr
} 
$[5,14]$ to nonconforming approximations of two-dimensional second order elliptic problems, where the equivalence between the exact error and the estimator on star, is based there on Helmholtz decomposition of the error, which is no more valid in general three dimensional geometries due to convexity requirement.

In this paper, an alternative approach for constructing an a posteriori error estimator for nonconforming approximation of scalar second order elliptic problem, based on the solution of local problems on stars, is given. We prove in general dimensions the efficiency and the reliability of this estimator, without saturation assumption. Moreover, explicit constants for transfer operator $([4,16])$ are given, which proves that this estimator is robust in suitable norms. The outline of the paper is as follows. In section 2 we introduce the functional framework and introduce the diffusionreaction problem with non nonconforming finite element approximation. In section three, we introduce the star-based a posteriori error estimator and perform the analysis for diffusion reaction problem. In section four, we extend the analysis to Stokes equation by adapting introduced arguments. Numerical results are given in section five to illustrate the good behavior and the efficiency of the given estimator on examples involving smooth and less-smooth solutions.

\section{Setting of the problem}

We consider the diffusion-reaction problem

$$
(P)\left\{\begin{array}{l}
-\Delta u+\sigma u=f \text { in } \Omega \\
u=0 \text { on } \Gamma:=\partial \Omega
\end{array}\right.
$$

where we assume that $\sigma \in L^{\infty}(\Omega)$ and $f \in L^{2}(\Omega)$, and $\Omega \subset \mathbb{R}^{d}, d=2,3$, is a simply connected polygonal domain. Let $\mathcal{T}_{h}$ be a family of conforming shape-regular triangulations of $\Omega$ by d-simplexes. We denote by $E_{I}$ the set of interior edges (faces) and by $E_{f}$ the set of all edges (faces) included in $\Gamma$. Let $V_{h}$ be the lowest order nonconforming Crouzeix-Raviart finite element space defined by

$$
V_{h}=\left\{v_{h} \in L^{2}(\Omega) ; \forall T \in \mathcal{T}_{h}, v_{h \mid T} \in P_{1}(T), \forall E \in E_{I}, \int_{E}\left[v_{h}\right]_{E} d \gamma=0 \text { and } \forall E \in E_{f}, \int_{E} v_{h} d \gamma=0\right\},
$$

where $[.]_{E}$ denotes the jump of the argument across $E$.

We denote by $\left\{x_{i}\right\}_{i \in \mathcal{N}}$ the set of all nodes of the triangulation $\mathcal{T}_{h}$. In the paper, by $i \in \mathcal{N}$ we will refer to the node $x_{i}$. For each $i \in \mathcal{N}, \phi_{i}$ denotes the canonical continuous piecewise linear basis function associated to $x_{i}$. The star $\omega_{i}$ is the interior relative to $\Omega$ of the support of $\phi_{i}$, and $h_{i}$ is the maximal size (diameters) of the elements constituting $\omega_{i}$. Finally, $\Gamma_{i}$ denotes the union of the edges (faces) touching $x_{i}$ that are contained in $\Omega$, and $\bar{\Gamma}_{i}$ the union of the edges (faces) touching $x_{i}$ that are contained in $\bar{\Omega}$. $h_{E}$ denotes the size (diameter) of an edge (face) E.

For each star $\omega_{i}, i \in \mathcal{N}$, we introduce the space $V\left(\omega_{i}\right)$ defined by

$$
V\left(\omega_{i}\right)=\left\{v \in H_{l o c}^{1}\left(\omega_{i}\right): \int_{\omega_{i}} v \phi_{i} d x=0\right\}, \quad \text { if } x_{i} \text { is an interior node, }
$$

and

$$
V\left(\omega_{i}\right)=\left\{v \in H_{l o c}^{1}\left(\omega_{i}\right): v=0 \text { on } \partial \omega_{i} \cap \Gamma\right\}, \quad \text { if } x_{i} \text { is a boundary node. }
$$

There exists a constant $C$, only depending on the minimum angle of the triangulation but independent of the star being considered, such that (see Prop. 2.4 of [11]) :

$$
\forall v \in V\left(\omega_{i}\right), \quad\|v\|_{0, \omega_{i}} \leq C h_{i}\left(\int_{\omega_{i}}|\nabla v|^{2} \phi_{i} d x\right)^{1 / 2} .
$$

We define the finite dimensional local spaces $\mathcal{P}^{2}\left(\omega_{i}\right)$ and $\mathcal{P}_{0}^{2}\left(\omega_{i}\right)$ as follows, 
Definition 1. For $i \in \mathcal{N}$, let $\mathcal{P}^{2}\left(\omega_{i}\right)$ denote the space of continuous piecewise quadratic functions on the star $\omega_{i}$ that vanish on $\partial \omega_{i}$. The space $\mathcal{P}_{0}^{2}\left(\omega_{i}\right)$ is defined by $\mathcal{P}_{0}^{2}\left(\omega_{i}\right)=\mathcal{P}^{2}\left(\omega_{i}\right) \cap V\left(\omega_{i}\right)$.

Let us introduce the usual $H^{1}$-norm on $\omega_{i}$,

$$
\|u\|_{1, \omega_{i}}^{2}=\|\nabla u\|_{0, \omega_{i}}^{2}+\|u\|_{0, \omega_{i}}^{2} .
$$

Let $v_{h} \in V_{h}$ be fixed. We denote by $\nabla_{h} v_{h}$ the vector field belonging to $\left(L^{2}(\Omega)\right)^{d}$, defined by

$$
\forall T \in \mathcal{T}_{h}, \quad \nabla_{h} v_{h}=\nabla v_{h} \text { on } T .
$$

Let $u_{h}^{N C} \in V_{h}$ be a solution of the nonconforming approximation problem:

$$
\left(P_{h}\right)^{N C} \quad \forall v_{h} \in V_{h} \cap H_{0}^{1}(\Omega), \quad a\left(u_{h}^{N C}, v_{h}\right):=\sum_{T \in \mathcal{T}_{h}} \int_{T}\left[\nabla u_{h}^{N C} \cdot \nabla v_{h}+\sigma u_{h}^{N C} v_{h}\right] d x=\int_{\Omega} f v_{h} d x .
$$

We will now turn to the construction of a $u_{h}^{C}$ belonging to $V_{h} \cap H_{0}^{1}(\Omega)$ such that

$$
\left\|u-u_{h}^{C}\right\|_{1, \Omega} \simeq \sum_{i \in \mathcal{N}}\left(\left\|u-u_{h}^{N C}\right\|_{1, \omega_{i}}^{2}\right)^{\frac{1}{2}} .
$$

\section{The star-based error estimate}

For each $i \in \mathcal{N}$, we consider the local problems :

$$
\left(P_{i}\right)\left\{\begin{array}{l}
\text { Find } \eta_{i} \in \mathcal{P}_{0}^{2}\left(\omega_{i}\right) \text { such that } \forall \mu_{i} \in \mathcal{P}_{0}^{2}\left(\omega_{i}\right), \\
\int_{\omega_{i}}\left(\nabla \eta_{i} . \nabla \mu_{i}\right) \phi_{i} d x=\int_{\omega_{i}} \nabla_{h} u_{h}^{N C} \cdot \nabla\left(\mu_{i} \phi_{i}\right) d x+\int_{\omega_{i}} \sigma u_{h}^{N C} \mu_{i} \phi_{i} d x-\int_{\omega_{i}} f \mu_{i} \phi_{i} d x .
\end{array}\right.
$$

Using Lax-Milgram Theorem, we can prove that each discrete problem $\left(P_{i}\right)$ admits a unique solution $\eta_{i}$.

Now we introduce the local error indicators,

$$
\forall i \in \mathcal{N}, \forall u_{h}^{N C} \in V_{h}, \quad E_{1, i}^{2}\left(u_{h}^{N C}\right)=\int_{\omega_{i}}\left|\nabla \eta_{i}\right|^{2} \phi_{i} d x
$$

and

$$
\forall i \in \mathcal{N}, \forall u_{h}^{N C} \in V_{h}, \quad E_{2, i}^{2}\left(u_{h}^{N C}\right)=\sum_{E \in \omega_{i}} h_{E}^{-1}\left\|\left[u_{h}^{N C}\right]_{E}\right\|_{0, E}^{2}
$$

\subsection{Upper bound}

We consider first the upper bound of the error without oscillation, and we step the process to the main theorem by the following intermediate lemmas.

The first lemma is an adaptation of arguments given in [11] and so the proof will be skipped.

Lemma 2. For all $i \in \mathcal{N}$, there exists an operator $\Pi_{i}: V\left(\omega_{i}\right) \longrightarrow \mathcal{P}_{0}^{2}\left(\omega_{i}\right)$, such that for any $v \in V\left(\omega_{i}\right)$ the following conditions hold:

1. For all edge (face) $E \subset \Gamma_{i}, \int_{E}\left(v-\Pi_{i} v\right) \phi_{i} d \gamma=0$.

2. Moreover, $\int_{\omega_{i}}\left(v-\Pi_{i} v\right) \phi_{i} d x=0$, if $x_{i}$ is an interior node.

3. $\left(\int_{\omega_{i}}\left|\nabla \Pi_{i} v\right|^{2} \phi_{i} d x\right)^{\frac{1}{2}} \leq C\left(\int_{\omega_{i}}|\nabla v|^{2} \phi_{i} d x\right)^{\frac{1}{2}}$. 
where $C$ is a positive constant only depending on the minimum angle of $\mathcal{T}_{h}$.

Lemma 3. For each $i \in \mathcal{N}$, each $v \in V\left(\omega_{i}\right)$ and $u_{h} \in V_{h}$, we have

$$
\int_{\omega_{i}} \nabla_{h} u_{h} \cdot \nabla\left(\left(\Pi_{i} v\right) \phi_{i}\right) d x=\int_{\omega_{i}} \nabla_{h} u_{h} \cdot \nabla\left(v \phi_{i}\right) d x .
$$

Proof. If we denote by $\left[\frac{\partial u_{h}}{\partial n_{E}}\right] \in P_{0}(E)$ the jump of the normal derivative across E, we have by applying Green formula and subsequently using the property 1 . of Lemma 2 ,

$$
\int_{\omega_{i}} \nabla_{h} u_{h} . \nabla\left(\left(\Pi_{i} v\right) \phi_{i}\right) d x=\sum_{E \subset \omega_{i}} \int_{E}\left[\frac{\partial u_{h}}{\partial n_{E}}\right]\left(\Pi_{i} v\right) \phi_{i} d \gamma=\sum_{E \subset \omega_{i}} \int_{E}\left[\frac{\partial u_{h}}{\partial n_{E}}\right] v \phi_{i} d \gamma
$$

Applying again Green formula yields the result.

Now we define the data oscillation by

$$
\operatorname{osc}(f)=\left(\sum_{i \in \mathcal{N}} h_{i}^{2}\left\|\left(f-f_{i}-\sigma u_{h}^{N C}\right) \phi_{i}^{\frac{1}{2}}\right\|_{0, \omega_{i}}^{2}\right)^{\frac{1}{2}},
$$

where $f_{i}=\frac{\int_{\omega_{i}} f \phi_{i} d x}{\int_{\omega_{i}} \phi_{i} d x}$ for $i$ interior nodes and 0 otherwise.

We have the following result about the a posteriori error estimate for any conforming approximation;

Theorem 4. Let $u_{h}^{N C} \in V_{h}$ be a solution of $\left(P_{h}^{N C}\right)$ and $u_{h}^{C} \in V_{h} \cap H_{0}^{1}(\Omega)$. There exists a positive constant $C$ only depending on the minimum angle of $\mathcal{T}_{h}$ such that

$$
\left\|u-u_{h}^{C}\right\|_{1, \Omega} \leq C\left[\left(\sum_{i \in \mathcal{N}} E_{1, i}^{2}\left(u_{h}^{N C}\right)\right)^{\frac{1}{2}}+\left(\sum_{i \in \mathcal{N}}\left\|u_{h}^{N C}-u_{h}^{C}\right\|_{1, \omega_{i}}^{2}\right)^{\frac{1}{2}}+o s c(f)\right] .
$$

Proof. Let $v$ be an element of $H_{0}^{1}(\Omega)$ and set $\tilde{v}:=\sum_{i \in \mathcal{N}} v_{i} \phi_{i}$, where $v_{i}=\frac{\int_{\omega_{i}} v \phi_{i} d x}{\int_{\omega_{i}} \phi_{i} d x}$ for interior nodes and 0 otherwise.

We have by adapting standard arguments used in the analysis of finite element approximation of finite approximation of elliptic problems and introducing $u_{h}^{N C}$,

$$
\left\|u-u_{h}^{C}\right\|_{1, \Omega} \leq C \sup _{v \in H_{0}^{1}(\Omega)} \frac{\left|a\left(u-u_{h}^{N C}, v\right)+a\left(u_{h}^{N C}-u_{h}^{C}, v\right)\right|}{\|v\|_{1, \Omega}} .
$$

Since $\tilde{v} \in V_{h} \cap H_{0}^{1}(\Omega), \quad a\left(u_{h}^{N C}-u, \tilde{v}\right)=0$. This gives,

$$
\begin{aligned}
a\left(u_{h}^{N C}-u, v\right) & =a\left(u_{h}^{N C}-u, v-\tilde{v}\right), \\
& =\sum_{i \in \mathcal{N}}\left[\int_{\omega_{i}} \nabla_{h} u_{h}^{N C} \cdot \nabla(v-\tilde{v}) d x+\int_{\omega_{i}} \sigma u_{h}^{N C}(v-\tilde{v}) d x-\int_{\omega_{i}} f(v-\tilde{v}) d x\right],
\end{aligned}
$$




$$
\begin{aligned}
& \text { Stating that } v-\tilde{v}=\sum_{i \in \mathcal{N}}\left(v-v_{i}\right) \phi_{i} \text {, and using } \sum_{i \in \mathcal{N}} \phi_{i}(x)=1 \text { gives } \\
& a\left(u_{h}^{N C}-u, v\right)=\sum_{i \in \mathcal{N}}\left[\int_{\omega_{i}} \nabla_{h} u_{h}^{N C} \cdot \nabla\left[\left(v-v_{i}\right) \phi_{i}\right] d x+\int_{\omega_{i}} \sigma u_{h}^{N C}\left(v-v_{i}\right) \phi_{i} d x-\int_{\omega_{i}} f\left(v-v_{i}\right) \phi_{i} d x\right] .
\end{aligned}
$$

Since $\left(v-v_{i}\right) \in V\left(\omega_{i}\right)$, adding and removing same quantities in the two last terms give

$$
\begin{aligned}
a\left(u_{h}^{N C}-u, v\right) & =\sum_{i \in \mathcal{N}}\left[\int_{\omega_{i}} \nabla_{h} u_{h}^{N C} \cdot \nabla\left[\Pi_{i}\left(v-v_{i}\right) \phi_{i}\right] d x+\int_{\omega_{i}} \sigma u_{h}^{N C} \Pi_{i}\left(v-v_{i}\right) \phi_{i} d x-\int_{\omega_{i}} f \Pi_{i}\left(v-v_{i}\right) \phi_{i} d x\right] \\
& -\sum_{i \in \mathcal{N}} \int_{\omega_{i}}\left(f-\sigma u_{h}^{N C}\right)\left(v-v_{i}-\Pi_{i}\left(v-v_{i}\right)\right) \phi_{i} d x .
\end{aligned}
$$

Using the definition of local problems $\left(P_{i}\right)$,

$$
a\left(u_{h}^{N C}-u, v\right)=\sum_{i \in \mathcal{N}}\left[\int_{\omega_{i}} \nabla \eta_{i} \cdot \nabla\left[\Pi_{i}\left(v-v_{i}\right)\right] \phi_{i} d x\right]-\sum_{i \in \mathcal{N}}\left[\int_{\omega_{i}}\left(f-\sigma u_{h}^{N C}\right)\left(v-v_{i}-\Pi_{i}\left(v-v_{i}\right)\right) \phi_{i} d x\right] .
$$

We now process successively with each term of the right-hand side. On one hand, using Cauchy-Schwarz and item 2. of Lemma 2 we have

$$
\begin{aligned}
\sum_{i \in \mathcal{N}}\left[\int_{\omega_{i}} \nabla \eta_{i} \cdot \nabla \Pi_{i}\left(v-v_{i}\right) \phi_{i} d x\right] & \leq\left(\sum_{i \in \mathcal{N}} \int_{\omega_{i}}\left|\nabla \eta_{i}\right|^{2} \phi_{i} d x\right)^{\frac{1}{2}}\left(\sum_{i \in \mathcal{N}} \int_{\omega_{i}}\left|\nabla \Pi_{i}\left(v-v_{i}\right)\right|^{2} \phi_{i} d x\right)^{\frac{1}{2}}, \\
& \leq C\left(\sum_{i \in \mathcal{N}} E_{1, i}^{2}\left(u_{h}^{N C}\right)\right)^{\frac{1}{2}}\left(\sum_{i \in \mathcal{N}} \int_{\omega_{i}}\left|\nabla\left(v-v_{i}\right)\right|^{2} \phi_{i} d x\right)^{\frac{1}{2}}, \\
& \leq C\left(\sum_{i \in \mathcal{N}} E_{1, i}^{2}\left(u_{h}^{N C}\right)\right)^{\frac{1}{2}}\|v\|_{1, \Omega} .
\end{aligned}
$$

On the other hand, since both of $\left(v-v_{i}\right)$ and $\Pi_{i}\left(v-v_{i}\right)$ belong to $V\left(w_{i}\right)$, using definition of $V\left(\omega_{i}\right)$ and coefficients $f_{i}$ give

$$
\sum_{i \in \mathcal{N}}\left[\int_{\omega_{i}}\left(f-\sigma u_{h}^{N C}\right)\left(v-v_{i}-\Pi_{i}\left(v-v_{i}\right)\right) \phi_{i} d x\right]=\sum_{i \in \mathcal{N}}\left[\int_{\omega_{i}}\left(f-f_{i}-\sigma u_{h}^{N C}\right)\left(v-v_{i}-\Pi_{i}\left(v-v_{i}\right)\right) \phi_{i} d x\right],
$$

Using Cauchy-Schwarz then inequality (2.1) and once more $\sum_{i \in \mathcal{N}} \phi_{i}(x)=1$, we get

$$
\begin{aligned}
\sum_{i \in \mathcal{N}}\left[\int_{\omega_{i}}\left(f-\sigma u_{h}^{N C}\right)\left(v-v_{i}-\Pi_{i}\left(v-v_{i}\right)\right) \phi_{i} d x\right] & \leq \operatorname{osc}(f)\left(\sum_{i \in \mathcal{N}} h_{i}^{-2}\left\|\left(v-v_{i}-\Pi_{i}\left(v-v_{i}\right)\right)\left(\phi_{i}\right)^{\frac{1}{2}}\right\|_{0, \omega_{i}}^{2}\right)^{\frac{1}{2}}, \\
& \leq \operatorname{Cosc}(f)\|v\|_{1, \Omega} .
\end{aligned}
$$

$C$ is a generic constant only depending on the minimum angle of triangulation.

Finally, summing up the different contributions in the estimate of $\left\|u-u_{h}^{C}\right\|_{1, \Omega}$ and using the continuity of $a(.,$.$) yield$ the result.

Summarizing the previous results gives the following result about the a posteriori error estimate for the nonconforming approximation:

Theorem 5. Let $u_{h}^{N C} \in V_{h}$ be a solution of $\left(P_{h}^{N C}\right)$ and $u_{h}^{C}$ be an arbitrary function of $\in V_{h} \cap H_{0}^{1}(\Omega)$. We have

$$
\left(\sum_{i \in \mathcal{N}}\left\|u-u_{h}^{N C}\right\|_{1, \omega_{i}}^{2}\right)^{\frac{1}{2}} \leq C\left[\left(\sum_{i \in \mathcal{N}}\left\|u_{h}^{N C}-u_{h}^{C}\right\|_{1, \omega_{i}}^{2}\right)^{\frac{1}{2}}+\left(\sum_{i \in \mathcal{N}} E_{1, i}^{2}\left(u_{h}^{N C}\right)\right)^{\frac{1}{2}}+\operatorname{osc}(f)\right],
$$

where $C$ only depends on the minimum angle of $\mathcal{T}_{h}$. 
In order to prove now the reliability of the estimator, we need the following lemma [10],

Lemma 6. There exists a linear operator $I: V_{h} \longrightarrow V_{h} \cap H_{0}^{1}(\Omega)$, satisfying the following estimate

$$
\forall u_{h}^{N C} \in V_{h}, \forall \omega_{i} \in \mathcal{T}_{h}, k=0,1,\left\|u_{h}^{N C}-I u_{h}^{N C}\right\|_{k, \omega_{i}} \leq C \sum_{E \in E_{I}, \bar{E} \cap \omega_{i} \neq \emptyset} h_{E}^{\frac{1}{2}-k}\left\|\left[u_{h}^{N C}\right]_{E}\right\|_{0, E},
$$

where $h_{E}$ is the diameter of face (edge) $E$.

\subsection{Lower bound}

In this section we prove a lower bound of the error without oscillation.

Theorem 7. Let $u_{h}^{N C} \in V_{h}$, there exist generic positive constant $C$ depending on the minimum angle of the triangulation such that, for any $i \in \mathcal{N}$,

$$
E_{1, i}\left(u_{h}^{N C}\right) \leq C\left\|u-u_{h}^{N C}\right\|_{1, \omega_{i}}
$$

and

$$
E_{2, i}\left(u_{h}^{N C}\right) \leq C\left(\sum_{i \in \mathcal{N}}\left\|u-u_{h}^{N C}\right\|_{1, \omega_{i}}^{2}\right)^{\frac{1}{2}} .
$$

Proof. We refer for a proof of second estimate to [10], and proceed with the first one. For each $i \in \mathcal{N}$, by definition of $E_{1, i}\left(u_{h}^{N C}\right)$ and taking test function $\mu_{i}=\eta_{i}$ in local problem $\left(P_{i}\right)$ give

$$
\begin{aligned}
E_{1, i}^{2}\left(u_{h}^{N C}\right) & =\int_{\omega_{i}}\left(\left|\nabla \eta_{i}\right|^{2} \phi_{i} d x\right), \\
& =\int_{\omega_{i}} \nabla u_{h}^{N C} \cdot \nabla\left(\eta_{i} \phi_{i}\right) d x+\int_{\omega_{i}}\left(\sigma u_{h}^{N C} \eta_{i}\right) \phi_{i} d x-\int_{\omega_{i}} f \eta_{i} \phi_{i} d x,
\end{aligned}
$$

Since $\left(\eta_{i} \phi_{i}\right) \in H_{0}^{1}\left(\omega_{i}\right)$, we have $\int_{\omega_{i}} \nabla u \cdot \nabla\left(\eta_{i} \phi_{i}\right) d x+\int_{\omega_{i}} \sigma u \eta_{i} \phi_{i} d x=\int_{\omega_{i}} f \eta_{i} \phi_{i} d x$. This gives

$$
\begin{aligned}
E_{1, i}^{2}\left(u_{h}^{N C}\right) & =\int_{\omega_{i}}\left(\nabla_{h} u_{h}^{N C}-\nabla u\right) \nabla\left(\eta_{i} \phi_{i}\right) d x+\int_{\omega_{i}}\left(\sigma\left(u_{h}^{N C}-u\right) \eta_{i} \phi_{i} d x,\right. \\
& =\int_{\omega_{i}}\left(\nabla_{h} u_{h}^{N C}-\nabla u\right) \nabla\left(\eta_{i}\right) \phi_{i} d x+\int_{\omega_{i}}\left(\nabla_{h} u_{h}^{N C}-\nabla u\right)\left(\eta_{i}\right) \nabla\left(\phi_{i}\right) d x+\int_{\omega_{i}}\left(\sigma\left(u_{h}^{N C}-u\right) \eta_{i} \phi_{i} d x .\right.
\end{aligned}
$$

Applying Cauchy-Schwarz inequality gives

$$
\begin{aligned}
E_{1, i}^{2}\left(u_{h}^{N C}\right) \leq & \left\|u-u_{h}^{N C}\right\|_{1, \omega_{i}} E_{1, i}\left(u_{h}\right)+\left\|u-u_{h}^{N C}\right\|_{1, \omega_{i}}\left\|\eta_{i}\right\|_{0, \omega_{i}}\left\|\phi_{i}\right\|_{W^{1, \infty}\left(w_{i}\right)} \\
& +\|\sigma\|_{L^{\infty}\left(\omega_{i}\right)}\left\|u-u_{h}^{N C}\right\|_{0, \omega_{i}}\left\|\eta_{i}\right\|_{0, \omega_{i}},
\end{aligned}
$$

$\phi_{i}$ being bounded in $\omega_{i}$. Now since $\eta_{i} \in V\left(\omega_{i}\right)$, using (2.1), we have

$$
\left\|\eta_{i}\right\|_{0, \omega_{i}} \leq C h_{i} E_{1, i}\left(u_{h}^{N C}\right) .
$$

Finally using the property $\left|\phi_{i}\right|_{W^{1, \infty}\left(\omega_{i}\right)} \leq \frac{C}{h_{i}}$, we get

$$
E_{1, i}\left(u_{h}^{N C}\right) \leq C\left(1+\|\sigma\|_{L^{\infty}(\Omega)} h_{i}\right)\left\|u-u_{h}^{N C}\right\|_{1, \omega_{i}} .
$$

which concludes the proof. 


\section{Extension to Stokes problem}

Let us now extend the ideas given above to the Stokes equations. We will define an error estimator for this problem and prove that it is equivalent with the energy error. Given a simply connected domain $\Omega \subset \mathbb{R}^{d} d=2,3$, we consider then the Stokes problem,

$$
(S P)\left\{\begin{aligned}
-\Delta u+\nabla p=f & \text { in } \Omega, \\
\operatorname{div} u=0 & \text { in } \Omega, \\
u=0 & \text { on } \Gamma=\partial \Omega,
\end{aligned}\right.
$$

where $f \in\left(L^{2}(\Omega)\right)^{d}$.

$V_{h}$ being defined in section 2, we set

$$
Q_{h}=\left\{q_{h} \in L_{0}^{2}(\Omega), q_{h \mid T} \in P_{0}(T), \forall T \in \omega_{i} \text { and } \omega_{i} \in \mathcal{T}_{h}\right\} .
$$

and consider the approximate solution $\left(u_{h}^{N C}, p_{h}\right) \in\left(V_{h}\right)^{d} \times Q_{h}$ defined by

$$
\left\{\begin{array}{l}
\forall v_{h} \in\left(V_{h}\right)^{d}, \quad \sum_{T \in \mathcal{T}_{h}}\left\{\int_{T} \nabla u_{h}^{N C}: \nabla v_{h} d x-\int_{T} p_{h} \operatorname{div} v_{h} d x\right\}=\int_{\Omega} f \cdot v_{h} d x, \\
\forall q_{h} \in Q_{h}, \quad \sum_{T \in \mathcal{T}_{h}} \int_{T} q \operatorname{div} u_{h}^{N C} d x=0
\end{array}\right.
$$

Note that the second equation means that for every $T \in \mathcal{T}_{h}, \operatorname{div}\left(u_{h}^{N C}{ }_{{ }_{T}}\right)=0$.

Let $v_{h} \in\left(V_{h}\right)^{d}$ be fixed. We define $\nabla_{h} v_{h}$ and $\operatorname{div}_{h} v_{h}$ by :

$$
\forall T \in \mathcal{T}_{h}, \quad \nabla_{h} v_{h}=\nabla v_{h} \text { on } T,
$$

and

$$
\forall T \in \mathcal{T}_{h}, \quad \operatorname{div}_{h} v_{h}=\operatorname{div} v_{h} \text { on } T .
$$

We introduce the following local problems :

$$
\left(S P_{i}\right)\left\{\begin{array}{l}
\text { Find } \epsilon_{i} \in\left(\mathcal{P}_{0}^{2}\left(\omega_{i}\right)\right)^{d} \text { such that } \\
\forall \mu_{i} \in\left(\mathcal{P}_{0}^{2}\left(\omega_{i}\right)\right)^{d}, \int_{\omega_{i}}\left(\nabla \epsilon_{i}: \nabla \mu_{i}\right) \phi_{i} d x=\int_{\omega_{i}} \nabla_{h} u_{h}^{N C}: \nabla\left(\mu_{i} \phi_{i}\right) d x \\
\quad-\int_{\omega_{i}} p_{h} \operatorname{div}\left(\mu_{i} \phi_{i}\right) d x-\int_{\omega_{i}}\left(f \cdot \mu_{i}\right) \phi_{i} d x .
\end{array}\right.
$$

It is obvious that these local problems admit unique solutions.

We introduce for all $i \in \mathcal{N}$ the three indicators,

$$
\eta_{1, i}\left(u_{h}^{N C}, p_{h}\right)=\left(\sum_{T \in \omega_{i}}\left\|\operatorname{div}_{h} u_{h}^{N C} \phi_{i}^{\frac{1}{2}}\right\|_{0, T}^{2}\right)^{\frac{1}{2}}
$$




$$
\begin{gathered}
\eta_{2, i}\left(u_{h}^{N C}, p_{h}\right)=\left(\int_{\omega_{i}}\left|\nabla \epsilon_{i}\right|^{2} \phi_{i} d x\right)^{\frac{1}{2}}, \\
\eta_{3}^{2}\left(u_{h}^{N C}, p_{h}\right)=\sum_{E \in E_{I}} h_{E}^{-1}\left\|\left[u_{h}^{N C}\right]_{E}\right\|_{0, E}^{2},
\end{gathered}
$$

and set the problem data oscillation,

$$
\operatorname{osc}(f)=\left(\sum_{i \in \mathcal{N}} h_{i}^{2}\left\|\left(f-f_{i}\right) \phi_{i}^{\frac{1}{2}}\right\|_{0, \omega_{i}}^{2}\right)^{\frac{1}{2}}
$$

where $f_{i}=\frac{\int_{\omega_{i}} f \phi_{i} d x}{\int_{\omega_{i}} \phi_{i} d x}$ for interior nodes, and $f_{i}=0$ otherwise.

As previously, we give the first lemma which proof is similar to Lemma 2 one.

Lemma 8. For each $i \in \mathcal{N}$, there exists an operator $\Pi_{i}:\left(V\left(\omega_{i}\right)\right)^{d} \longrightarrow\left(\mathcal{P}_{0}^{2}\left(\omega_{i}\right)\right)^{d}$, such that for any $v \in\left(V\left(\omega_{i}\right)\right)^{d}$ the following assumptions hold:

1. For all edge $E \subset \Gamma_{i}, \int_{E}\left(v-\Pi_{i} v\right) \phi_{i} d \gamma=0$,

2. For all $v \in\left(V\left(\omega_{i}\right)\right)^{d}$ and $v_{h} \in\left(V_{h}\right)^{d}, \int_{\omega_{i}} \nabla_{h} v_{h}: \nabla\left(\left(\Pi_{i} v-v\right) \phi_{i}\right) d x=0$,

3. For all $q_{h} \in Q_{h}, \int_{\omega_{i}} q_{h} \operatorname{div}\left(v-\Pi_{i} v\right) \phi_{i} d x=0$.

The following theorem gives the a posteriori error estimate for the nonconforming finite element approximation of Stokes problem solution.

Theorem 9. There exists a positive constant $C$ depending on the minimum angle of the triangulation such that :

$$
\left(\sum_{i \in \mathcal{N}}\left\|u-u_{h}^{N C}\right\|_{1, \omega_{i}}^{2}\right)^{\frac{1}{2}}+\left\|p-p_{h}\right\|_{0, \Omega} \leq C\left\{\left[\left(\sum_{i \in \mathcal{N}} \eta_{1, i}^{2}+\eta_{2, i}^{2}\right)+\eta_{3}^{2}\right]^{\frac{1}{2}}+\operatorname{osc}(f)\right\}
$$

where, for more readability, we have skipped the arguments of $\eta_{1, i}, \eta_{2, i}$ and $\eta_{3}$, and so will be done in the sequel.

Proof. Since $\left(I u_{h}^{N C}, p_{h}\right) \in\left(H_{0}^{1}(\Omega)\right)^{d} \times L_{0}^{2}(\Omega)$, by standard finite element analysis arguments we state

$$
\left(\sum_{i \in \mathcal{N}}\left\|u-I u_{h}^{N C}\right\|_{1, \omega_{i}}^{2}\right)^{\frac{1}{2}}+\left\|p-p_{h}\right\|_{0, \Omega} \leq C \sup _{\left.\left.(v, q) \in\left(H_{0}^{1}(\Omega)\right)\right)^{d} \times L_{0}^{2}(\Omega)\right)} \frac{\left|a((u, p) ;(v, q))-a\left(\left(I u_{h}^{N C}, p_{h}\right) ;(v, q)\right)\right|}{|v|_{1, \Omega}+\|q\|_{0, \Omega}}
$$

where $a(. ;$.$) is defined by$

$$
\forall(u, p),(v, q) \in\left(H_{0}^{1}(\Omega)\right)^{d} \times L_{0}^{2}(\Omega), a((u, p) ;(v, q))=\int_{\Omega} \nabla u: \nabla v d x-\int_{\Omega} p \operatorname{div} v d x+\int_{\Omega} q \operatorname{div} u d x .
$$

then

$a((u, p) ;(v, q))-a\left(\left(I u_{h}^{N C}, p_{h}\right) ;(v, q)\right)=\int_{\Omega}\left(\nabla u-\nabla I u_{h}^{N C}\right): \nabla v d x-\int_{\Omega}\left(p-p_{h}\right) \operatorname{div} v d x+\int_{\Omega} q \operatorname{div}{ }_{h}\left(u-I u_{h}^{N C}\right) d x$. 
On one hand, since $\operatorname{div} u=0$ on $\Omega, \sum_{i} \phi_{i}(x)=1$ and $\phi_{i}$ being bounded, we have

$$
\left|\int_{\Omega} q \operatorname{div}\left(u-I u_{h}^{N C}\right) d x\right| \leq C \sum_{\omega_{i} \in \mathcal{T}_{h}}\|q\|_{0, \omega_{i}}\left\|\operatorname{div} I u_{h}^{N C} \phi_{i}^{\frac{1}{2}}\right\|_{0, \omega_{i}} .
$$

By virtue of Lemma 6 with $k=1$, we have

$$
\begin{aligned}
\forall \omega_{i} \in \mathcal{T}_{h},\left\|\operatorname{div} I u_{h}^{N C} \phi_{i}^{\frac{1}{2}}\right\|_{0, \omega_{i}} & \leq\left\|\operatorname{div}_{h} u_{h}^{N C} \phi_{i}^{\frac{1}{2}}\right\|_{0, \omega_{i}}+\left\|\left(\operatorname{div}_{h} u_{h}^{N C}-\operatorname{div} I u_{h}^{N C}\right) \phi_{i}^{\frac{1}{2}}\right\|_{0, \omega_{i}}, \\
& \leq\left\|\operatorname{div}_{h} u_{h}^{N C}\right\|_{0, \omega_{i}}+C \sum_{E \in E_{I}} h^{-\frac{1}{2}}\left\|\left[u_{h}^{N C}\right]_{E}\right\|_{0, E} .
\end{aligned}
$$

Summing up the contributions, using given indicators definitions and the inequality $\sum_{i} \alpha_{i} \beta_{i} \leq\left(\sum_{i} \alpha_{i}^{2}\right)^{1 / 2}\left(\sum_{i} \beta_{i}^{2}\right)^{1 / 2}$, we get

$$
\left|\int_{\Omega} q \operatorname{div}\left(u-I u_{h}^{N C}\right) d x\right| \leq C\left(\sum_{\omega_{i} \in \mathcal{T}_{h}}\|q\|_{0, \omega_{i}}^{2}\right)\left(\sum_{i \in \mathcal{N}} \eta_{1, i}^{2}+\eta_{3}^{2}\right)^{\frac{1}{2}} .
$$

On the other hand,

$$
\begin{aligned}
A:=\int_{\Omega}(\nabla u- & \left.\nabla I u_{h}^{N C}\right): \nabla v d x-\int_{\Omega}\left(p-p_{h}\right) \operatorname{div} v d x=\sum_{\omega_{i} \in \mathcal{T}_{h}}\left[\int_{\omega_{i}}\left(\nabla u-\nabla_{h} u_{h}^{N C}\right): \nabla v d x\right. \\
& \left.+\int_{\omega_{i}}\left(\nabla_{h} u_{h}^{N C}-\nabla I u_{h}^{N C}\right): \nabla v d x-\int_{\omega_{i}}\left(p-p_{h}\right) \operatorname{div} v d x\right] \\
= & -\sum_{\omega_{i} \in \mathcal{T}_{h}} \int_{\omega_{i}}\left(\nabla_{h} u_{h}^{N C}\right): \nabla v d x+\int_{\omega_{i}} p_{h} \operatorname{div} v d x+\int_{\omega_{i}} f \cdot v d x+\int_{\omega_{i}}\left(\nabla_{h} u_{h}^{N C}-\nabla I u_{h}^{N C}\right): \nabla v d x
\end{aligned}
$$

Introducing the field $\tilde{v} \in\left(V_{h}\right)^{d} \cap\left(H_{0}^{1}(\Omega)\right)^{d}$, in the same manner as in proof of theorem 4 in order to involve $\left(v-v_{i}\right) \in$ $\left(V\left(\omega_{i}\right)\right)^{d}$ and use item 2. of Lemma 8 we get

$$
\begin{aligned}
A & =-\sum_{\omega_{i} \in \mathcal{T}_{h}} \int_{\omega_{i}}\left(\nabla_{h} u_{h}^{N C}\right): \nabla\left[\Pi_{i}\left(v-v_{i}\right) \phi_{i}\right] d x+\int_{\omega_{i}} p_{h}\left[\operatorname{div} \Pi_{i}\left(v-v_{i}\right) \phi_{i}\right] d x+\int_{\omega_{i}} f \cdot \Pi_{i}\left(v-v_{i}\right) \phi_{i} d x \\
& +\int_{\omega_{i}} p_{h}\left[\operatorname{div}_{h}\left(v-v_{i}-\Pi_{i}\left(v-v_{i}\right)\right) \phi_{i}\right] d x+\int_{\omega_{i}}\left(\nabla_{h} u_{h}^{N C}-\nabla I u_{h}^{N C}\right): \nabla v d x+\int_{\omega_{i}} f \cdot\left(v-v_{i}-\Pi_{i}\left(v-v_{i}\right)\right) \phi_{i} d x,
\end{aligned}
$$

Adapting arguments used in Theorem 4 and using successively item 3. of Lemma 8, definition of local problems $\left(S P_{i}\right)$, Lemma 6 with $k=1$, we get

$$
A \leq\left\{\left(\sum_{i \in \mathcal{N}} \eta_{2, i}^{2}+\eta_{3}^{2}\right)^{\frac{1}{2}}+o s c(f)\right\}|v|_{1, \Omega}
$$

Summing up the contributions gives,

$$
\left|a((u, p) ;(v, q))-a\left(\left(I_{h}^{N C}, p_{h}\right) ;(v, q)\right)\right| \leq\left(\sum_{i \in \mathcal{N}} \eta_{1, i}^{2}+\eta_{3}^{2}\right)^{\frac{1}{2}}\|q\|_{0, \Omega}+\left\{\left(\sum_{i \in \mathcal{N}} \eta_{2, i}^{2}+\eta_{3}^{2}\right)^{\frac{1}{2}}+o s c(f)\right\}|v|_{1, \Omega}
$$

Finally stating

$$
\sum_{\omega_{i} \in \mathcal{T}_{h}}\left\|u-u_{h}^{N C}\right\|_{1, \omega_{i}}^{2} \leq \sum_{\omega_{i} \in \mathcal{T}_{h}}\left\|u-I u_{h}^{N C}\right\|_{1, \omega_{i}}^{2}+\sum_{\omega_{i} \in \mathcal{T}_{h}}\left\|u_{h}^{N C}-I u_{h}^{N C}\right\|_{1, \omega_{i}}^{2} \leq C \eta_{3}^{2}+\sum_{\omega_{i} \in \mathcal{T}_{h}}\left\|u-I u_{h}^{N C}\right\|_{1, \omega_{i}}^{2},
$$

yield the result. 


\section{Efficiency of the estimator:}

Theorem 10. $\forall \omega_{i} \in \mathcal{T}_{h}$, we have the following inequalities,

$$
\begin{gathered}
\left\|\operatorname{div}_{h} u_{h}^{N C}\right\|_{0, \omega_{i}} \leq C\left\|u-u_{h}^{N C}\right\|_{1, \omega_{i}}, \\
\eta_{2, i} \leq C\left(\left\|u-u_{h}^{N C}\right\|_{1, \omega_{i}}+\left\|p-p_{h}\right\|_{0, \omega_{i}}\right)
\end{gathered}
$$

and

$$
\eta_{3} \leq C\left\|u-u_{h}^{N C}\right\|_{1, \omega_{i}} .
$$

Proof. The first inequality is obvious and the third one has already been proved in Theorem 7 . So we proceed with the second estimation. Using the definition indicator and local problems $\left(S P_{i}\right)$,

$$
\begin{aligned}
\eta_{2, i}^{2}\left(u_{h}^{N C}, p_{h}\right) & =\int_{\omega_{i}}\left(\left|\nabla \epsilon_{i}\right|^{2} \phi_{i} d x\right), \\
& =\int_{\omega_{i}} \nabla_{h} u_{h}^{N C}: \nabla\left(\epsilon_{i} \phi_{i}\right) d x-\int_{\omega_{i}} p_{h} \operatorname{div}\left(\epsilon_{i} \phi_{i}\right) d x-\int_{\omega_{i}} f . \epsilon_{i} \phi_{i} d x,
\end{aligned}
$$

As in section 3, since $\left(\epsilon_{i} \phi_{i}\right) \in\left(H_{0}^{1}\left(\omega_{i}\right)\right)^{d}$, we can state

$$
\begin{aligned}
\eta_{2, i}^{2}\left(u_{h}^{N C}, p_{h}\right) & =\int_{\omega_{i}}\left(\nabla_{h} u_{h}^{N C}-\nabla u\right): \nabla\left(\epsilon_{i} \phi_{i}\right) d x-\int_{\omega_{i}}\left(p_{h}-p\right) \operatorname{div}\left(\epsilon_{i} \phi_{i}\right) d x, \\
& =\int_{\omega_{i}}\left(\nabla_{h} u_{h}^{N C}-\nabla u\right): \nabla\left(\epsilon_{i}\right) \phi_{i} d x+\int_{\omega_{i}}\left(\nabla_{h} u_{h}^{N C}-\nabla u\right)\left(\epsilon_{i}\right): \nabla\left(\phi_{i}\right) d x-\int_{\omega_{i}}\left(p_{h}-p\right) \operatorname{div}\left(\epsilon_{i} \phi_{i}\right) d x,
\end{aligned}
$$

and following same steps as in proof of Theorem 7, we retrieve the second estimation.

\section{Numerical experiments}

\section{Diffusion reaction example:}

For the numerical illustration of the efficiency of the error estimator and the based adaption process, we consider a model problem with homogeneous data on the computational domain $[0,1]^{2}$, with the source term $f$ given by the exact solution,

$$
u=x y(x-1)(y-1) e^{-100(x-0.5)^{2}-100(y-0.117)^{2}},
$$

which presents sharp curvature in the vicinity of point $(0.5,0.117)$, and we perform a nonconforming finite element discretization on it. Successive iterations of adaptive mesh are represented in Figure 1. Computed and Exact solution are given in Figure 2, where the scaling of the height is the same for both pictures. 

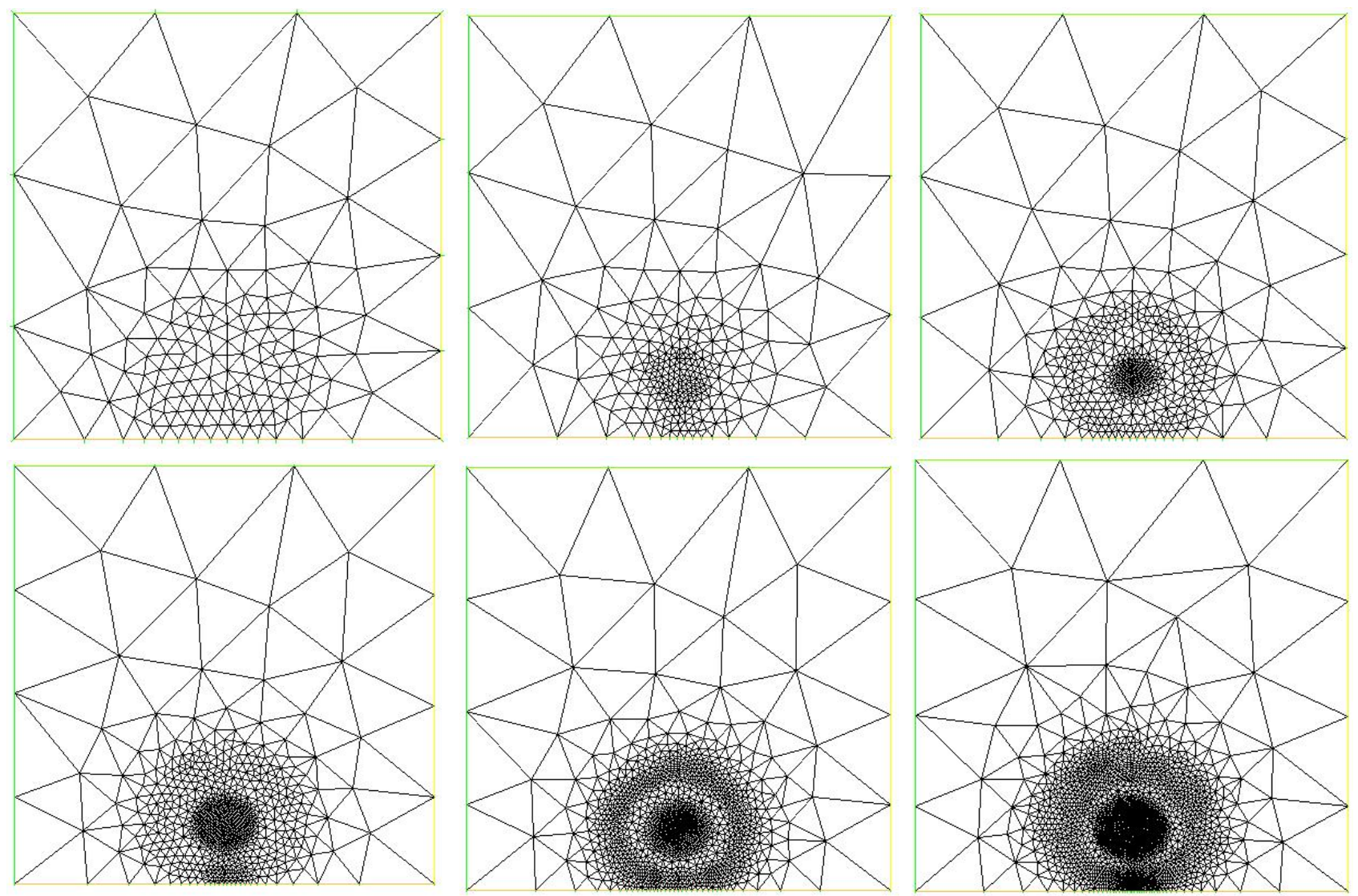

Figure 1: Adaptive mesh refinement using the error indicator.

Table 1 and Figure 3 give the evolution of the error indicator value and the error solution versus the number of degrees of freedom (ndof). We notice that the estimator and the error have analogous behavior, and the estimator under-estimates the energy norm error. Figure 3 illustrates quasi-optimality of the estimator, the dashed line of slope $(-1 / 2)$ showing a numerical (ndof) ${ }^{(-1 / 2)}$ asymptotic decay of the error estimator.

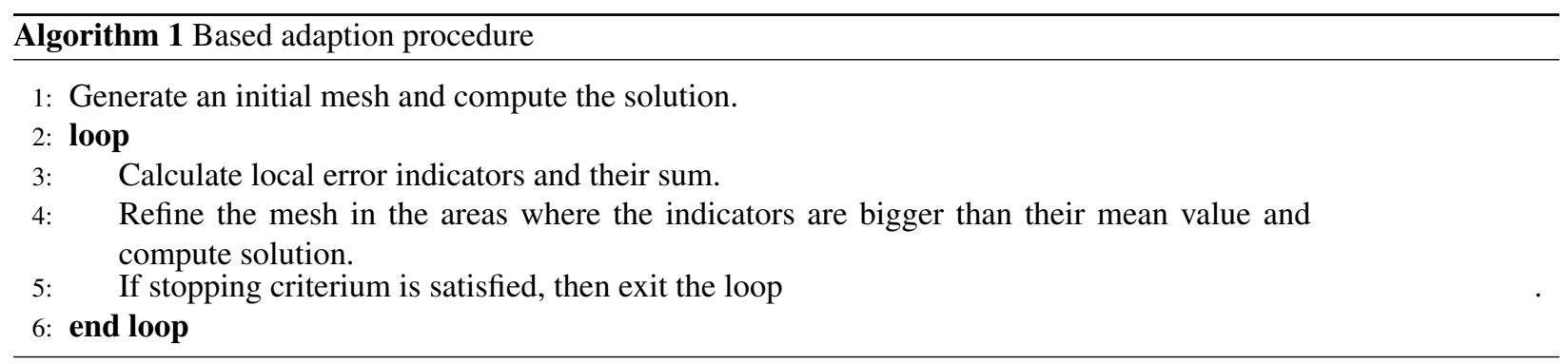



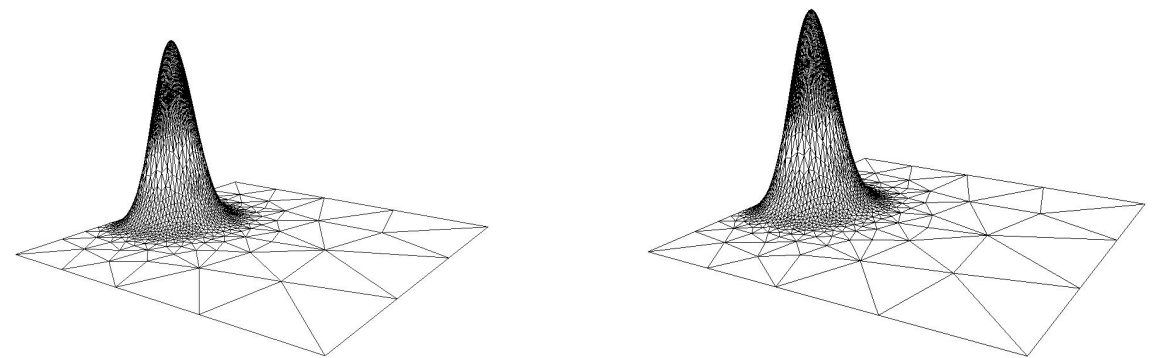

Figure 2: Computed solution (left) and exact solution (right) for diffusion reaction example (same scaling).

\begin{tabular}{|c|c|c|}
\hline ndof & Error indicator & $\left\|u-u_{h}^{N C}\right\|$ \\
\hline 449 & $3.4773 \mathrm{e}-001$ & $1.6271 \mathrm{e}-001$ \\
762 & $2.5113 \mathrm{e}-001$ & $1.1980 \mathrm{e}-001$ \\
1408 & $1.7055 \mathrm{e}-001$ & $9.4704 \mathrm{e}-002$ \\
5065 & $8.8906 \mathrm{e}-002$ & $5.3381 \mathrm{e}-002$ \\
9843 & $6.5920 \mathrm{e}-002$ & $3.6450 \mathrm{e}-002$ \\
19576 & $4.7111 \mathrm{e}-002$ & $2.8596 \mathrm{e}-002$ \\
38653 & $3.5282 \mathrm{e}-002$ & $1.9497 \mathrm{e}-002$ \\
77469 & $2.5526 \mathrm{e}-002$ & $1.5080 \mathrm{e}-002$ \\
153644 & $1.8019 \mathrm{e}-002$ & $1.0170 \mathrm{e}-002$ \\
\hline
\end{tabular}

Table 1: Error and indicator values for diffusion reaction problem.

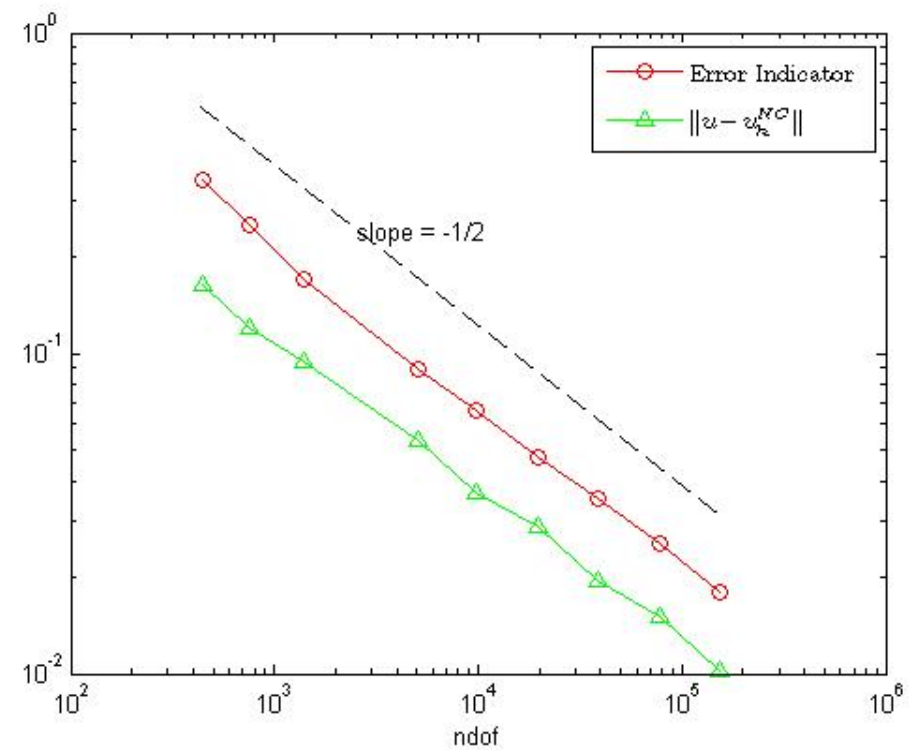

Figure 3: Decay of error indicator and energy error. The dashed line has slope of $-1 / 2$.

\section{Stokes problem example with analytic smooth solution :}

We consider the test case proposed par Bercovier and Engelman [18], defined on the unit square $[0,1]^{2}$ as follows,

$$
v(x, y)=-256 x^{2}(x-1)^{2} y(y-1)(2 y-1)
$$




$$
\begin{gathered}
u(x, y)=\left[\begin{array}{c}
v(x, y) \\
-v(x, y)
\end{array}\right] \\
p(x, y)=\left(x-\frac{1}{2}\right) \cdot\left(y-\frac{1}{2}\right) \\
f(x, y)=\left[\begin{array}{c}
-\nu v(x, y)+\left(y-\frac{1}{2}\right) \\
\nu v(x, y)+\left(x-\frac{1}{2}\right)
\end{array}\right]
\end{gathered}
$$

We perform nonconforming finite element discretization on it, and we report on Figure 4, Figure 5 and Figure 6 a sequence of adapted meshes using the proposed refinement indicators and corresponding computed velocity and pressure respectively.
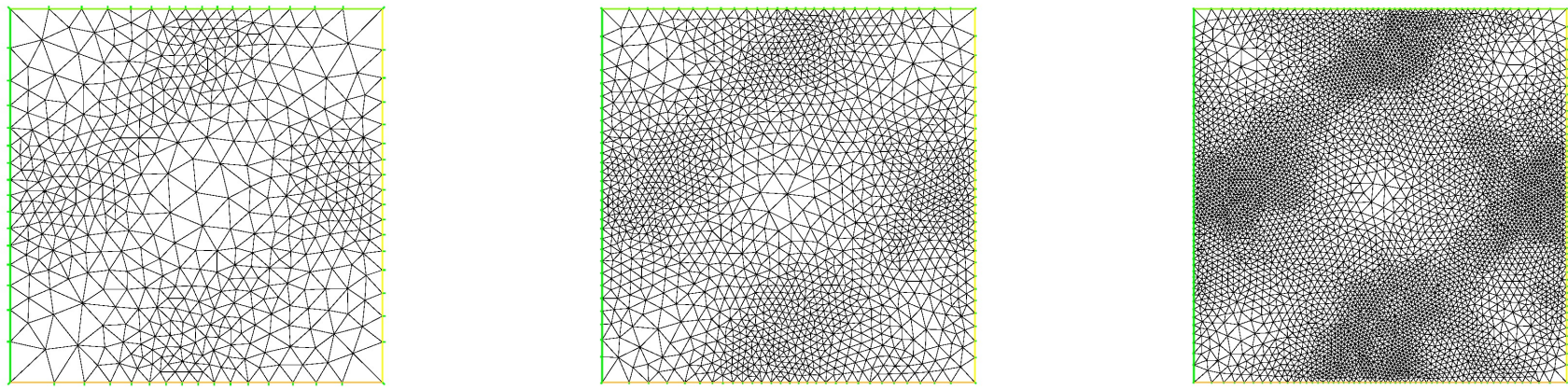

Figure 4: Adaptive mesh refinement using the error indicator.
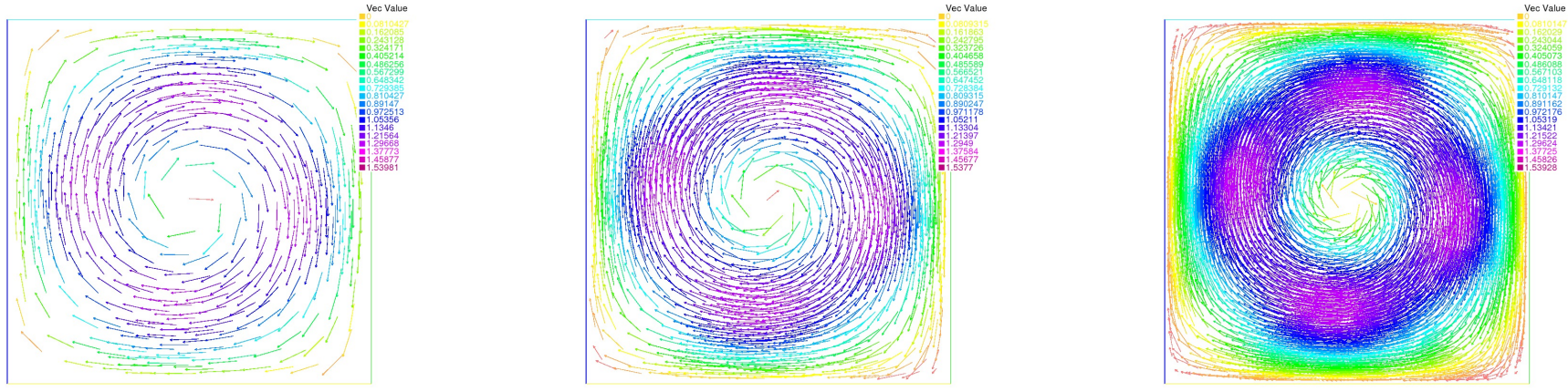

Figure 5: Adaptive computed velocity using the error indicator.

\section{Lid-driven cavity problem example:}

The two-dimensional Stokes driven cavity problem has been thoroughly studied in numerous references (eg. [19]). The main difficulty of this problem comes from the discontinuity of the velocity boundary data at corners. The problem configuration corresponds to a flow in a square cavity $[0,1]^{2}$. The top of the cavity moves from left to right, imparting motion to the fluid via the no-slip boundary condition, $\mathbf{u}=(1,0)$ on the top. The velocity on all other boundaries is zero, $\mathbf{u}=(0,0)$. We perform nonconforming finite element discretization on it, and we give below a sequence of adaptive meshes. Furthermore, we present the corresponding approximate velocity and pressure contour lines. 

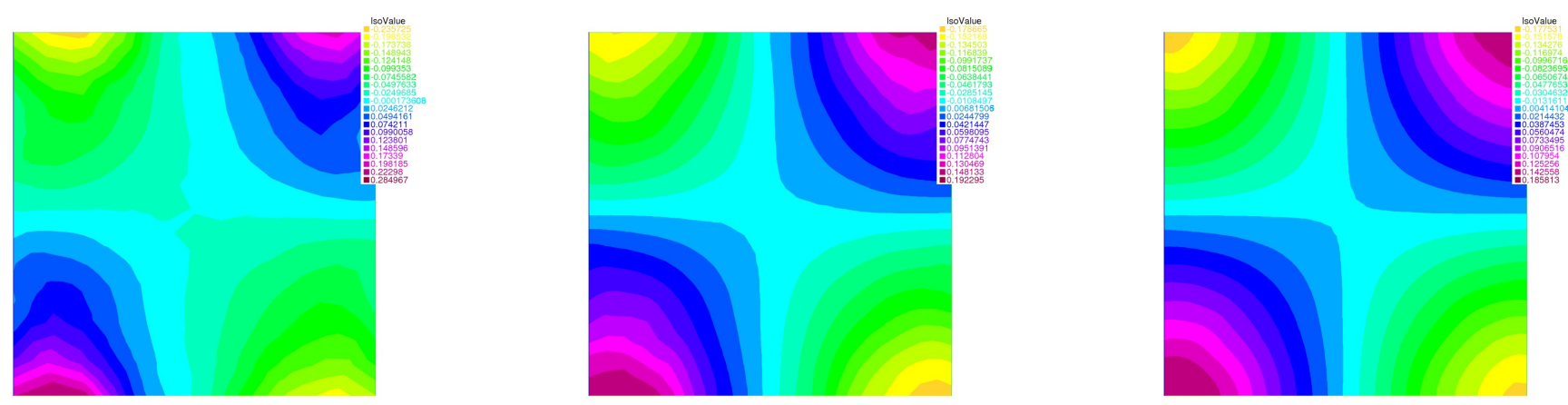

Figure 6: Adaptive computed pressure using the error indicator.
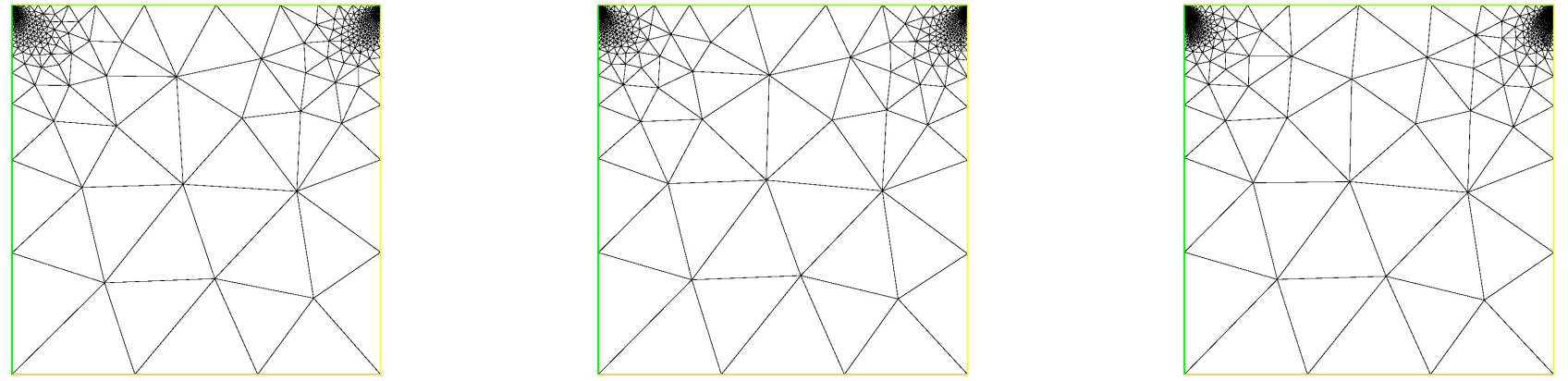

Figure 7: Error-indicator based refined meshes for Lid-driven Cavity problem.
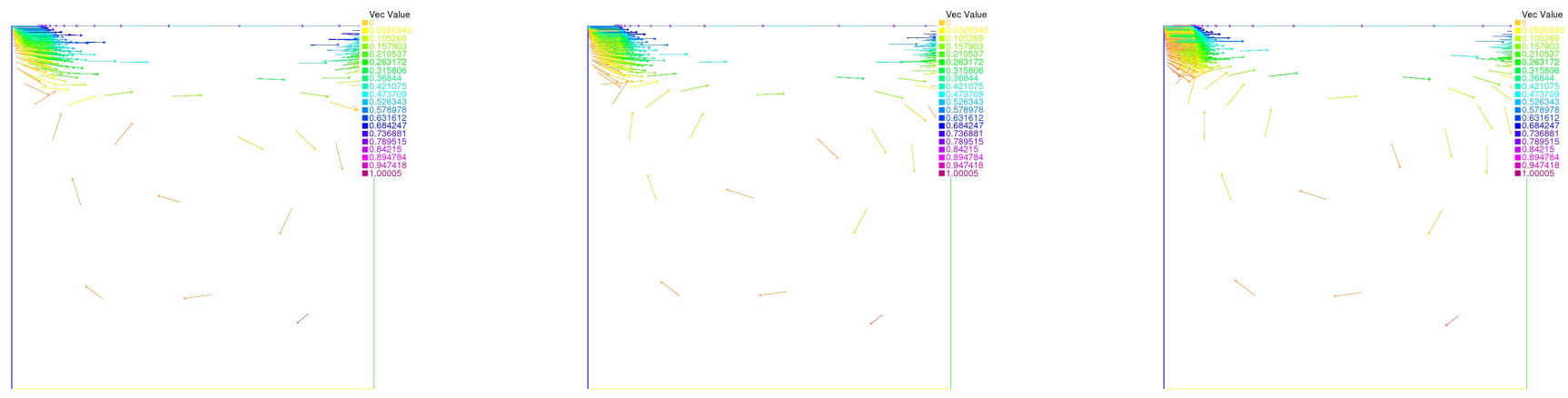

Figure 8: Adaptive computed velocity of Lid-driven Cavity problem.

\section{Conclusion}

We presented and analyzed an a posteriori error estimator for nonconforming approximations of reaction diffusion and Stokes equations. The construction of this so-called star-based error estimator is based on the solution of local subproblems. We proved that it is equivalent to the energy error up to a data oscillation, without requiring Helmholtz decomposition of the error nor saturation assumption. The proof is valid in general space dimensions. Two-dimensional numerical experiments illustrated the good behavior and confirmed the quasi-optimal predicted asymptotic rate of decay of this error estimator. 

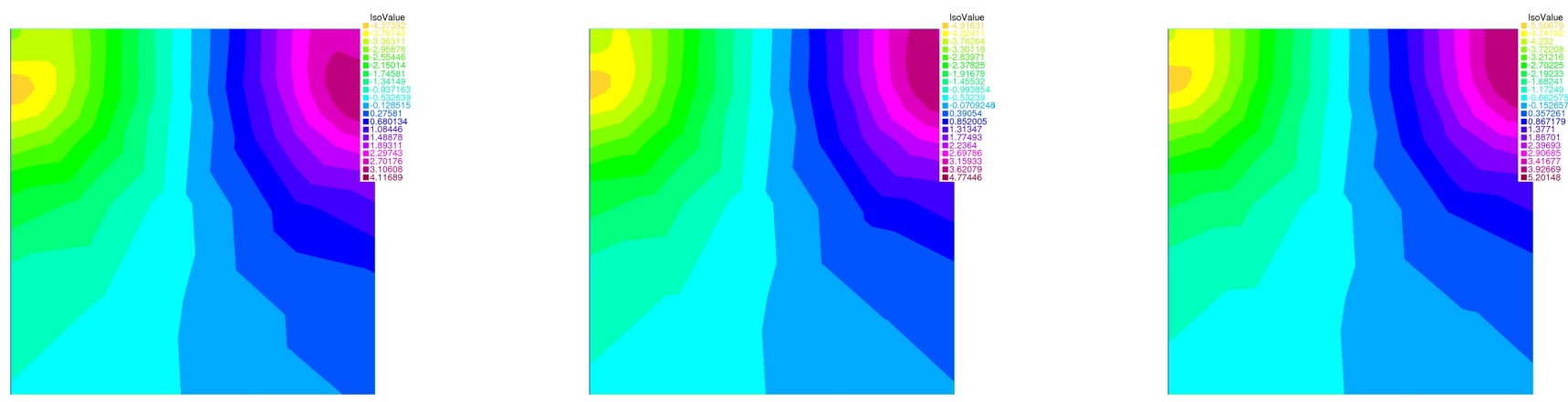

Figure 9: Adaptive computed pressure of Lid-driven Cavity problem.

\section{Acknowledgement}

This work is supported in part by HydroMed Project, and CNRST (Projet d'établissement, Université Hassan $1^{e r}$ Settat, Ministère de l'enseignement supérieur, Maroc).

These numerical simulations have been carried out using the software FreeFem++ developed by Frédéric Hecht and O. Pironneau : http://www.freefem.org/.

\section{References}

[1] B. Achchab, A. Agouzal, A. El Fatini and A. Souissi. Robust hierarchical a posteriori error estimates for stabilized convection-diffusion problem. Numer. Methods Partial Differential Eq.. doi: 10.1002/num.21696 (2012).

[2] A. Agouzal. A posteriori error estimator for nonconforming finite element methods. Appl. Math. Lett, 7(5), 1017-1033 (1994).

[3] A. Agouzal. A posteriori error estimator for finite element discretizations of quasi-Newtonian Stokes flows. Inter. J. of P. and Appl. Math., Vol 2, 2, 221-239 (2005).

[4] A. Agouzal. A posteriori error estimator for nonconforming approximation. Inter. J. of P. and Appl. Math., Vol 5, $1,77-85$ (2008).

[5] A. Agouzal and K. Allali. Local problem error estimator for nonconforming finite element approximations. Inter. J.of P. and Appl. Math., Vol 9, 4, 451-467 (2003).

[6] R.E. Bank and A. Weiser. Some a posteriori error estimators for elliptic partial differential equations. Math. Comp., 44, 283-301 (1985).

[7] R.E. Bank and D.Bruno Welfert. A posteriori error estimates for the Stokes problem. SIAM J. Num. Anal., 28, 591-623 (1991).

[8] E. Dari, R. Durán and C Padra. Error estimators for nonconforming finite element approximations of the Stokes problem. Math. Comp., Vol 64, 211, 1017-1033 (1995).

[9] E. Dari, R. Durán, C Padra and V. Vampa. A posteriori error estimator for nonconforming finite element methods M2AN, Vol 30, N 4, 385-400 (1996).

[10] O. A. Karakashian and F. Pascal. A posteriori error estimates for discontinuous Galerkin approximation of second-order elliptic problems. SIAM J. Num. Anal. 41, 6, 2374-2399 (2003). 
[11] P. Morin, R.H. Nochetto and K.G. Siebert. Local Problems on Stars: A Posteriori Error Estimators, Convergence, and Performance. Math. Comp., Vol 72, 243, 1067-1097 (2003).

[12] F. Nobile A posteriori error estimates for the finite element approximation of the Stokes problem. ICES Report 03-13, The University of Texas at Austin (2003).

[13] R.H. Nochetto. Removing the saturation assumption in a posteriori error analysis. Rend., Sci. Mat. Appl. A, 127, 6782 (1993).

[14] N. Parés, P. Díez and A. Huerta. Subdomain-based flux-free a posteriori error estimators. Comp. Meth. Appl. Mech. Eng., Vol. 195, Issues 4-6, 297-323 (2006).

[15] S. Prudhomme, F. Nobile, L. Chamoin and J.T. Oden. Analysis of a subdomain-based error estimator for finite element approximations of elliptic problems. Num. Meth. Part. Diff. Equ. Vol. 20, Issue 2, 165-192 (2004).

[16] F. Schieweck. A general transfer operator for arbitrary finite element spaces. Otto-von-Guericke-Universitt, Fakultt fr Mathematik, Preprint No. 25 (2000).

[17] R. Verfürth. A review of a posteriori error estimation and adaptive mesh-refinement techniques. Teubner Skripten zur Numerik, B.G. Teubner Stuttgart (1996).

[18] M. Bercovier and M. Engelman. A finite Element for the Numerical Solution of Viscous Incompressible Flows. J. of Comp. Physics, 30, 181-201 (1979).

[19] P.N. Shankar. The eddy structure in Stokes flow in a cavity. J. Fluid. Mech., 250, 371383 (1993). 